\title{
NONLINEAR MULTIGRID INVERSION
}

\author{
Seungseok Oh, Adam B. Milstein, Charles A. Bouman, and Kevin J Webb \\ School of Electrical and Computer Engineering, Purdue University \\ West Lafayette, Indiana 47907, USA
}

\begin{abstract}
In this paper, we propose a general framework for nonlinear multigrid inversion applicable to any inverse problem in which the forward model can be naturally represented at differing resolutions. In multigrid inversion, the problem is adjusted to be solved at each resolution by using the solutions at both finer and coarser resolutions. To do this, we formulate a consistent set of coarse scale cost functionals to ultimately reduce the finest scale one. At each resolution, both the forward model and inverse problems are discretized at the lower resolution; thus reducing computation. Our simulation results for the application of optical diffusion tomography indicate the potential for fast and robust convergence.
\end{abstract}

\section{INTRODUCTION}

A large class of image processing problems depends on the solution of inverse problems. To solve inverse problems, most algorithms work by performing all computations using a fixed discretization grid, but fixed-grid algorithms tend to have slow convergence for large nonlinear inverse problems. In some new imaging modalities, such as optical diffusion tomography (ODT), electrical impedance tomography, thermal wave imaging, and microwave imaging, the forward models are described by partial differential equations (PDEs). These applications present particularly challenging inverse problems because the forward model is highly nonlinear and computationally demanding to evaluate. In $[1,2]$, nonlinear multigrid optimization algorithms were used to reduce the computation required to solve the ODT inverse problem. However, a disadvantage of this approach is that it depended on successive linearization of the forward model. Related techniques have been independently proposed for the application of nonlinear multigrid methods to other optimization problems [3].

In this paper we propose a method we call multigrid inversion that is applicable to a wide variety of inverse problems. The multigrid inversion algorithm results from the application of recursive multigrid techniques to optimization arising from inverse problems. The method works by dynamically adjusting the cost functionals at different scales so that they are consistent with, and ultimately reduce, the finest scale cost functional.

A key innovation in our approach is that the resolution of both the forward and inverse models are varied at different grid resolutions. This makes our method particularly well suited to the solution of inverse problems with PDE forward models for a number of reasons: (1) The computation is dramatically reduced by using coarse grids to solve both the forward model PDE and its inversion; (2) the coarse grid forward model is computed using a dis-

This work was supported by the National Science Foundation under contract CCR-0073357. cretized version of the true PDE, thereby preserving its nonlinear characteristics; (3) a wide variety of linear or nonlinear fixed-grid optimization methods can be used for solving the inverse problem at each grid.

\section{COST FUNCTION}

Let $y$ be a (real or complex) measurement vector, and let $x$ be a finite dimensional vector representing the unknown quantity, in our case an image, to be reconstructed. The objective of inverse problems is to compute $x$ from $y$. This inversion can be reduced to an optimization problem. In this paper, we use a cost functional [2]

$$
c(x)=\frac{P}{2} \log \|y-f(x)\|_{\Lambda}^{2}+S(x)
$$

where $f(x)$ is the forward model which represents the computed means of the measurements given the image $x, \Lambda$ is a positive definite weight matrix, $P$ is the dimensionality of the measurement (i.e. the length of $y$ in real-valued data, or twice its length in complex-valued data), $S(x)$ is a stabilizing functional used to regularize the inverse, and $\|w\|_{\Lambda}^{2}=w^{H} \Lambda w$. Note that the logarithm in (1) results from incorporating the automatic estimate of the measurement noise as in [2]. However, our method is equally applicable to the case when a quadratic norm is used without the logarithm.

\section{NONLINEAR MULTIGRID INVERSION}

Once the cost functional of $(1)$ is formulated, the inverse is computed by minimizing the cost function with respect to $x$. In this section, we derive the basic multigrid inversion algorithm for solving the minimization of (1).

Let $x^{(0)}$ denote the finest grid image, and let $x^{(q)}$ be a coarse resolution representation of $x^{(0)}$ with a grid sampling period of $2^{9}$ times the finest grid sampling period. To obtain a coarser resolution image $x^{(q+1)}$ from a finer resolution image $x^{(q)}$, we use the relation $x^{(q+1)}=I_{(q)}^{(q+1)} x^{(q)}$, where $I_{(q)}^{(q+1)}$ is a linear decimation matrix. We use $I_{(q+1)}^{(q)}$ to denote the corresponding linear interpolation matrix.

We define a cost functional, with a form analogous to that of (1), but with quantities indexed by the scale $q$ and appending an additional linear correction term, as

$$
\begin{aligned}
c^{(q)}\left(x^{(q)}\right)= & \frac{P}{2} \log \left\|y^{(q)}-f^{(q)}\left(x^{(q)}\right)\right\|_{\Lambda}^{2} \\
& +S^{(q)}\left(x^{(q)}\right)-r^{(q)} x^{(q)}
\end{aligned}
$$


where $r^{(q)}$ is a row vector used to adjust the functional's gradient. At the finest scale, all quantities take on their fine scale values and $r^{(q)}=0$, so that $c^{(0)}\left(x^{(0)}\right)=c(x)$. Notice that the forward model $f^{(q)}(\cdot)$ and the stabilizing functional $S^{(q)}(\cdot)$ are both evaluated at scale $q$, and thus evaluation of the forward model at low resolu* tion substantially reduces computation due to the reduced number of variables. The quantity $y^{(q)}$ denotes an adjusted measurement vector at scale $q$. The stabilizing functional at each scale is fixed and chosen to best approximate the fine scale functional.

In the remainder of this section, we explain how the cost functionals at each scale can be matched to produce a consistent solution. Specifically, our objective is to derive recursive expressions for the quantities $y^{(q)}$ and $r^{(q)}$ that match the cost functionals at fine and coarse scales. We do not describe in this paper how to adjust the stabilizing functional. For an example of the choice of coarse scale stabilizing functional, see [2], where the generalized Gaussian Markov random field (GGMRF) model is used for the image prior model in maximum a posteriori (MAP) estimation.

Let $x^{(q)}$ be the current solution at grid $q$. We would like to improve this solution by first performing iterations of fixed-grid optimization at the coarser grid $q+1$, and then using this result to correct the finer grid solution. This coarse grid update is given by

$$
\tilde{x}^{(q+1)} \leftarrow \text { Fixed_Grid_Update }\left(I_{(q)}^{(q+1)} x^{(q)}, c^{(q+1)}(\cdot)\right)
$$

where $\tilde{x}^{(q+1)}$ is the updated value, and the operator

Fixed_Grid_Update $\left(x_{\text {init }}, c(\cdot)\right)$ is any fixed-grid update algorithm designed to reduce the cost functional $c(\cdot)$ starting with the initial value $x_{\text {init }}$. In (3), the initial condition $I_{(q)}^{(q+1)} x^{(q)}$ is formed by decimating $x^{(q)}$. We may now use this result to update the finer grid solution. We do this by interpolating the change in the coarser scale solution as

$$
\hat{x}^{(q)} \leftarrow x^{(q)}+I_{(q+1)}^{(q)}\left(\tilde{x}^{(q+1)}-I_{(q)}^{(q+1)} x^{(q)}\right) .
$$

Ideally, the new solutions $\tilde{x}^{(q)}$ should be at least as good as the old solution $x^{(q)}$. Specifically, we would like

$$
c^{(q)}\left(\tilde{x}^{(q)}\right) \leq c^{(q)}\left(x^{(q)}\right) .
$$

However, this may not be the case if the cost functionals are not consistent. In fact, for a naively chosen set of cost functionals, the coarse scale correction could easily move the solution away from the optimum.

This problem of inconsistent cost functionals is eliminated if the fine and coarse scale cost functionals are equal within an additive constant. This means we would like

$$
\begin{aligned}
& c^{(q+1)}\left(\tilde{x}^{(q+1)}\right) \\
\cong & c^{(q)}\left(x^{(q)}+I_{(q+1)}^{(q)}\left(\tilde{x}^{(q+1)}-I_{(q)}^{(q+1)} x^{(q)}\right)\right)+\text { const }
\end{aligned}
$$

to hold for all values of $\tilde{x}^{(q+1)}$. Our objective is then to choose a coarse scale cost functional which matches the fine cost functional as described in (6). We do this by the proper selection of $y^{(q+1)}$ and $r^{(q+1)}$. First, we enforce the condition that the initial error between the forward model and measurements be the same at the coarse and fine scales, giving

$$
y^{(q+1)}-f^{(q+1)}\left(I_{(q)}^{(q+1)} x^{(q)}\right)=y^{(q)}-f^{(q)}\left(x^{(q)}\right) .
$$

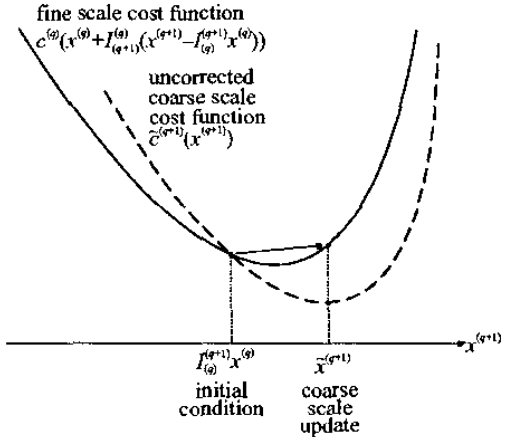

(a)

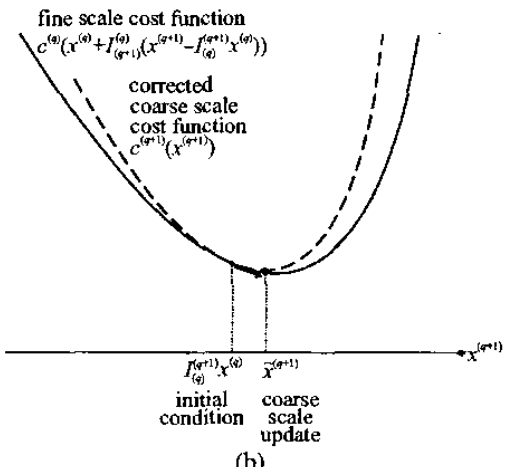

(b)

Fig. 1. The role of adjustment term $r^{(q+1)} x^{(q+1)}:$ (a) Inconsistent update (b) Consistent update

This yields the update for $y^{(q+1)}$

$$
y^{(q+1)} \leftarrow y^{(q)}-\left[f^{(q)}\left(x^{(q)}\right)-f^{(q+1)}\left(I_{(q)}^{(q+1)} x^{(q)}\right)\right] .
$$

Intuitively, the term in the bracket compensates for the forward model mismatch between resolutions.

Next, we use the condition introduced in [1,2] to enforce the condition that the gradients of the coarse and fine cost functionals be equal at the current values of $x^{(q)}$ and $x^{(q+1)}=I_{(q)}^{(q+1)} x^{(q)}$. More precisely, we enforce the condition that

$$
\left.\nabla c^{(q+1)}\left(x^{(q+1)}\right)\right|_{\left.x^{(q+1)}=I_{(q)}^{(q+1)}\right)_{x}(q)}=\nabla c^{(q)}\left(x^{(q)}\right) I_{(q+1)}^{(q)}
$$

This condition is essential to assuring that the optimum solution is a fixed point of the multigrid inversion algorithm [2], and is illustrated graphically in Fig. 1. In Section 4, we will also show how this condition can be used along with other assumptions to ensure monotone convergence of the multigrid inversion algorithm.

The equality of (9) can be enforced at the current value $x^{(q)}$ by choosing

$$
\begin{aligned}
r^{(q+1)} \leftarrow & \left.\nabla \tilde{e}^{(q+1)}\left(x^{(q+1)}\right)\right|_{x^{(q+1)}=I_{(q)}^{(q+1)} x^{(q)}} \\
& -\nabla c^{(q)}\left(x^{(q)}\right) I_{(q+1)}^{(q)},
\end{aligned}
$$

where $\tilde{c}^{(q)}(\cdot)$ is an approximate cost functional defined by

$$
\tilde{c}^{(q)}\left(x^{(q)}\right)=\frac{P}{2} \log \left\|y^{(q)}-f^{(q)}\left(x^{(q)}\right)\right\|_{\Lambda}^{2}+S^{(q)}\left(x^{(q)}\right) .
$$




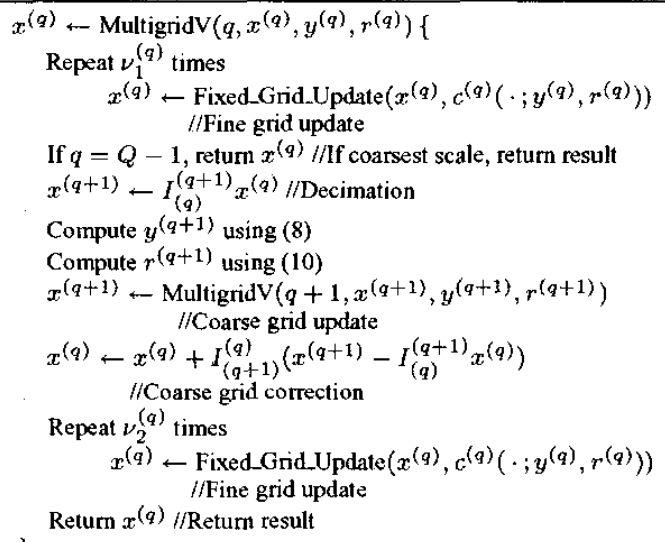

Fig. 2. Pseudo-code of the subroutine for the Multigrid-V inversion. The constant $Q$ is the total number of scales used.

The multigrid-V algorithm [4] is obtained by applying this two-grid algorithm recursively in resolution, as shown in the pseudocode in Fig. 2. After initialization of $r^{(0)} \leftarrow 0$ and $y^{(0)} \leftarrow$ $y$, we can then minimize (1) through iterative application of the MultigridV(.) subroutine at resolution 0. During each iteration, the multigrid-V algorithm moves from the finest to the coarsest resolutions, and subsequently moves back to the finest resolution. In Fig 2, we use the notation $c^{(q+1)}\left(x^{(q+1)} ; y^{(q+1)}, r^{(q+1)}\right)$ to make the dependency on $y^{(q+1)}$ and $r^{(q+1)}$ explicit.

\section{CONVERGENCE OF MULTIGRID INVERSION}

Multigrid inversion can be viewed as a method to simplify a potentially expensive optimization by temporarily replacing the original cost functional by a lower resolution one. In fact, there is a large class of optimization methods which depend on the use of so-called surrogate functionals, or functional substitution methods to speed or simplify optimization. A classic example of a surrogate functional is the Q-function used in the expectation-maximization (EM) algorithm [5]. More recently, the surrogate functional approach was applied to tomography problems in a manner that allowed parallel updates of pixels in the computation of penalized maximum likelihood (ML) reconstructions [6].

The application of surrogate functionals used in multigrid inversion is unique in that the substituting functional is at a coarser scale and therefore has an argument of lower dimension. As with traditional approaches, the surrogate functional should be designed to guarantee monotone convergence of the original cost functional. In the case of the multigrid algorithm, a sequence of optimization functionals at varying resolutions should be designed so that the entire multigrid update decreases the finest resolution cost.

Figure 1 graphically illustrates the use of surrogate functionals in multigrid inversion. Figure 1(a) shows the case in which the gradients of the fine scale and coarse scale (i.e. surrogate) functions are different at the initial value. In this case, the surrogate functional can not upper bound the value of the fine scale functional, and the updated value may actually increase the fine grid cost functional's value. Figure 1(b) illustrates the case in which the gradients of the two functionals are matched. In this case, a properly chosen coarse scale functional can upper bound the fine scale functional, and the coarse scale update is guaranteed to reduce the fine scale cost.

The concepts illustrated in Fig. 1 can be formalized into conditions that guarantee that each iteration of the multigrid algorithm reduces the fine grid cost functional. In this case, we say that the multigrid algorithm is monotone. The following theorem, proved in [7], gives a set of conditions which guarantee that the multigrid inversion algorithm is monotone.

Theorem: (Multigrid Monotone Convergence)

For $0 \leq q<Q-1$, define the functional $\xi^{(q+1)}: \mathbb{R}^{N^{(q+1)}} \rightarrow \mathbb{R}$

$$
\begin{aligned}
\xi^{(q+1)}\left(x^{(q+1)}\right)= & \tilde{c}^{(q+1)}\left(x^{(q+1)}\right) \\
& -\tilde{c}^{(q)}\left(x^{(q)}+I_{(q+1)}^{(q)}\left(x^{(q+1)}-I_{(q)}^{(q+1)} x^{(q)}\right)\right),
\end{aligned}
$$

where $N^{(q+1)}$ is the number of voxels in $x^{(q+1)}, I R$ is the set of real numbers, and the functions $\tilde{c}^{(q)}(\cdot)$ and $\tilde{c}^{(q+1)}(\cdot)$ are continuously differentiable. Assume that the following conditions are satisfied:

1. The fixed-grid update is monotone for $0 \leq q<Q$.

2. $\xi^{(q)}(\cdot)$ is convex on $\mathbb{R}^{N^{(q)}}$ for $0<q<Q$.

3. The adjustment vector $r^{(q+1)}$ is given by (10) for $0 \leq q<Q$.

4. $\nu_{1}^{(q)}+\nu_{2}^{(q)} \geq 1$ for $0 \leq q<Q$.

Then, the multigrid algorithm of Fig. 2 is monotone for $c^{(0)}(\cdot)$.

The conditions 1,3 , and 4 of the Theorem are easily satisfied for most problems. However, the difficulty lies in satisfying condition 2, convexity of $\xi^{(q)}(\cdot)$ for $q>0$. If the eigenvalues of the Hessian of $\xi^{(q)}(\cdot)$ are lower-bounded, the convexity condition can be satisfied by adding a convex term, such as $\gamma\left\|x^{(q)}\right\|^{2}$, to $\tilde{c}^{(q)}(\cdot)$ for $q>0$, where $\gamma$ is a sufficiently large constant. However, addition of such a term tends to slow convergence by making the coarse scale corrections too conservative.

When the forward model is given by a PDE, it can be difficult or impossible to verify or guarantee the convexity condition of 2 . Nonetheless, the theorem still gives insight into the convergence behavior of the algorithm; and in Section 5.2 we will show that empirically, for the difficult problem of ODT, the convergence of the multigrid algorithm is monotone in all cases, even without the addition of any convex terms.

\section{APPLICATION TO OPTICAL DIFFUSION TOMOGRAPHY}

\subsection{Optical diffusion tomography}

Optical diffusion tomography (ODT) is a method for determining spatial maps of optical properties from several boundary measurements of light transmitted through a highly scattering medium $[1,2]$. In frequency domain ODT, the measured modulation envelope $y$ of the photon flux is used to reconstruct the image $x$, i.e. the absorption coefficient $\mu_{a}$ and diffusion coefficient $D$ at each discretized grid point. The forward model is highly nonlinear because it is described by the solution to the diffusion equation where the unknowns are the coefficients of the PDE.

To estimate the image $x$, we use an independent Gaussian shot noise model and the generalized Gaussian Markov random field (GGMRF) image prior model in a Bayesian framework [2, 1]. Incorporating the automatic estimate of the measurement noise, the 


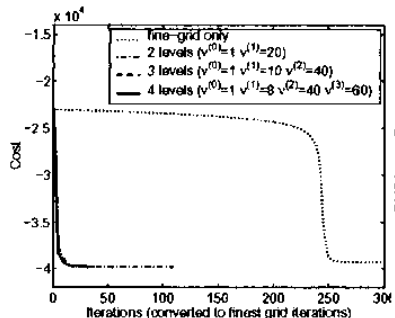

(a)

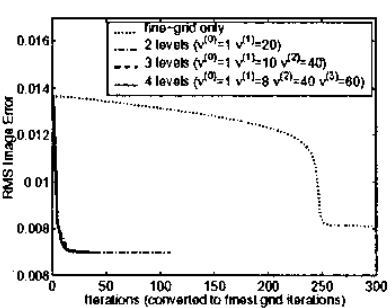

(b)
Fig. 3. Convergence of (a) cost function and (b) RMS image error when reconstructions were initialized with average values.

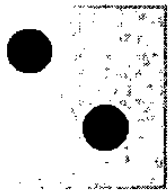

(a)

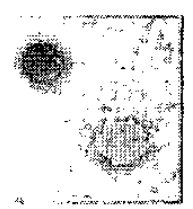

(b)

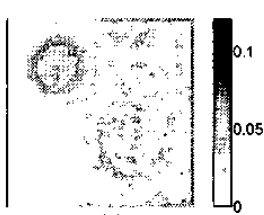

(c)
Fig. 4. Cross-sections on the center plane in the $z$-direction : (a) true phantom, (b) 4 level multigrid reconstruction with 19.35 iterations, and (c) fixed-grid reconstruction with 270 iterations.

resulting cost functional is of the same form as (1), and we can use the multigrid inversion algorithm developed in Section 3 to solve the required optimization problem.

\subsection{Numerical Results}

We examined the performance of the nonlinear multigrid inversion algorithm for the ODT problem. We used a cubic tissue phantom formed by two spherical $\mu_{a}$ inhomogeneities embedded in a background value of $\mu_{a}$ which varied linearly from $0.01 \mathrm{~cm}^{-1}$ to $0.04 \mathrm{~cm}^{-1}$ along the $x$-direction. The diffusion coefficient $D$ was homogeneous with $D=0.03 \mathrm{~cm}$. Eight sources and nine detectors were located on each face. All source-detector pairs were used, except those on the same face of the cube. Gaussian shot noise was added to the data, and the average signal-to-noise ratio for sources and detectors on opposite faces was $35 \mathrm{~dB}$.

For simplicity, we reconstructed only absorption coefficients. All comparisons of fixed-grid and multigrid inversion algorithms were made at $65 \times 65 \times 65$ resolution. For fixed-grid updates at each scale, we used the ICD algorithm. (See [7] for details.) In order to make fair comparisons of computational speed, we scaled the number of iterations for all methods into units of single fixedgrid iterations at the finest scale.

For the first experiment, all algorithms were initialized with the average values of the true phantom. Figure 3 shows that the multigrid algorithms converged in only 20 iterations, while the fixed algorithm required 270 iterations. The multigrid algorithms converged to slightly lower values of the cost $(-39833$ to -39763$)$ than the fixed-grid algorithm (-39392), and the root-mean-square image error for the multigrid reconstructions $(0.0069$ to 0.007$)$ was lower than the fixed algorithm (0.0081). In Fig. 4, the image quality of multigrid reconstruction was significantly better than the fixed-grid reconstruction.

To investigate the sensitivity of convergence with respect to initialization, we performed reconstructions with a poor initial estimate, i.e. a value of 1.75 times the true phantom's average value.

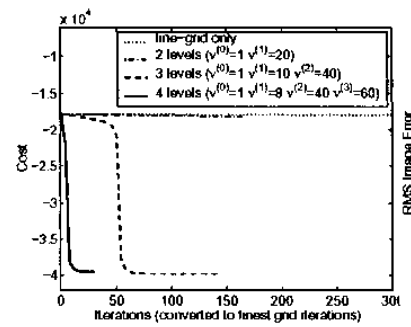

(a)

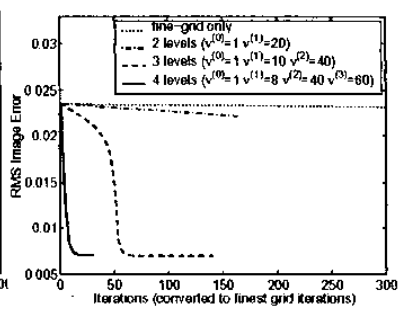

(b)
Fig. 5. Convergence of (a) cost function and (b) RMS image error when reconstructions were initialized with a poor initial guess.

Figure 5 shows that the three and four level multigrid algorithms converged rapidly. In particular, the four level multigrid algorithm converged almost as rapidly as it did when initialized with the true phantom's average value. The fixed-grid algorithm changed very little from the initial estimatc even after 300 iterations, and the two grid algorithm progressed slowly. These results suggest that higher level multigrid algorithms are necessary to overcome the effects of a poor initial estimate.

\section{CONCLUSIONS}

We have proposed a multigrid inversion algorithm which is particularly well suited for nonlinear inverse problems with forward models given by the solution to a PDE. Experimental results for the ODT application have shown the potential for very large computational savings and robust convergence with a range of initialization conditions.

\section{REFERENCES}

[1] J. C. Ye, C. A. Bouman, R. P. Millane, and K. J. Webb, "Nonlinear multigrid optimization for Bayesian diffusion tomography," in Proc. of IEEE Int'l Conf. on Image Proc., Kobe, Japan, October 25-28 1999.

[2] J. C. Ye, C. A. Bouman, K. J. Webb, and R. P. Millane, "Nonlinear multigrid algorithms for Bayesian optical diffusion tomography," IEEE Trans. on Image Processing, vol. 10, no. 6, pp. 909-922, June 2001.

[3] R. M. Lewis and S. G. Nash, "A multigrid approach to the optimization of systems governed by differential equations," in 8-th ALAA/USAF/ISSMO Symp. Multidisciplinary Analysis and Optimization, Long Beach, CA, 2000.

[4] W. L. Briggs, V.E. Henson, and S. F. McCormick, A Multigrid Tutorial, $2 n d E d$., Society for Industrial and Applied Mathematics, Philadelphia, 2000.

[5] L. E. Baum and T. Petrie, "Statistical inference for probabilistic functions of finite state Markov chains," Ann. Math. Statistics, vol. 37, pp. 1554-1563, 1966.

[6] A.R. De Pierro, "A modified expectation maximization algorithm for penalized likelihood estimation in emission tomography," IEEE Trans. on Medical Imaging, vol. 14, no. 1, pp. 132-137, March 1995.

[7] S. Oh, A. B. Milstein, C. A. Bouman, and K. J. Webb, "A general framework for nonlinear multigrid inversion," Technical Report TR-ECE-03-04, School of Electrical and Computer Engineering, Purdue University, 2003. 